\title{
Research on Applications of Shenmai Injection on Hypertensive ICH Combined with Cerebrocardiac Syndromes
}

\author{
Gang Yang1*, Jianju Feng², Shaojun Yang1, Junjie Lv¹, Donghai Yuan', Chenbing Wang1, \\ Feng Ding ${ }^{1}$, Chao Gu ${ }^{1}$, Gaofeng Shao ${ }^{1}$ \\ ${ }^{1}$ Department of Neurosurgery, Zhuji Affiliated Hospital of Shaoxing University, Shaoxing, China \\ ${ }^{2}$ Radiology Department, Zhuji Affiliated Hospital of Shaoxing University, Shaoxing, China \\ Email: *tekeyang@sina.com
}

How to cite this paper: Yang, G., Feng, J.J., Yang, S.J., Lv, J.J., Yuan, D.H., Wang, C.B., Ding, F., Gu, C. and Shao, G.F. (2021) Research on Applications of Shenmai Injection on Hypertensive ICH Combined with Cerebrocardiac Syndromes. Journal of Biosciences and Medicines, 9, 79-85.

https://doi.org/10.4236/jbm.2021.95008

Received: April 29, 2021

Accepted: May 23, 2021

Published: May 26, 2021

Copyright $\odot 2021$ by author(s) and Scientific Research Publishing Inc. This work is licensed under the Creative Commons Attribution International License (CC BY 4.0).

http://creativecommons.org/licenses/by/4.0/

(c) $\underset{\mathrm{By}}{\text { (i) Open Access }}$

\begin{abstract}
Objective: This study is to observe the therapeutic effects of Shenmai injection on patients with hypertensive ICH and cerebrocardiac syndromes as well as its effects on prognosis. Methods: A total of 72 patients with severe hypertensive ICH and cerebrocardiac syndromes were selected and divided into the control group and the experimental group (Shenmai injection group) randomly, 36 patients in each group. The control group was treated by conventional therapy, while the experimental group was administrated by intravenous drip of the mixture of $50 \mathrm{ml}$ Shenmai injection and $250 \mathrm{ml} 5 \%$ glucose injection based on the treatment of the control group, once everyday, 2 weeks continuously. Results: Myocardial enzyme indexes of two groups at $3 \mathrm{~d}, 7 \mathrm{~d}$ and $14 \mathrm{~d}$ were observed (Table 1). Myocardial enzyme index of the experimental group was improved quickly and significantly. Myocardial enzyme data of two groups at $3 \mathrm{~d}, 7 \mathrm{~d}$ and $14 \mathrm{~d}$ were $\mathrm{P}<0.05$. According to the electrocardiographic examinations results, ECG anomalies of the experimental group at $7 \mathrm{~d}$ and $14 \mathrm{~d}$ were also improved quickly $(\mathrm{P}<0.05)$. There are no evident anomalies in GCS scores of two groups at 7d, but GCS score of the experimental group at $14 \mathrm{~d}$ of the medication was significantly higher than that of the control group $(\mathrm{P}<0.05)$. Conclusions: Shenmai injection has some therapeutic effects on cerebral injuries and myocardial damages. It can treat brain and heart simultaneously, realize the goal of addressing both symptoms and root causes, and improve prognosis of patients with severe hypertensive ICH.
\end{abstract}

\section{Keywords}

Hypertensive ICH, Cerebrocardiac Syndromes, Shenmai Injection 


\section{Introduction}

Intracerebral hemorrhage (ICH) can threaten life and physical health of the aged group seriously due to its high morbidity, high fatality rate and high disability rate in clinics. Moreover, most survivors have different degrees of dysneuria. ICH brings heavy life and psychological burdens to the society, family and the patients. Cerebrocardiac syndromes are clinical syndromes which are developed upon cardiac dysfunction or myocardial disorder caused by serious diseases of central nervous system, and they are also called brain-derived cardiovascular dysfunction. Cerebrocardiac syndromes are similar with acute myocardial infarction (MI) or myocardial ischemia and arrhythmia. There are mutual influences and crosslinking relations between heart and brain. So far, there's no systematic therapeutic method. Only few studies on the combination of severe hypertensive ICH and cerebrocardiac syndromes have been reported in China. It has not attracted enough attentions in clinics, accompanied with high rate of missed diagnosis and misdiagnosis rate. Moreover, most antiarrhythmic drugs have poor therapeutic effects to cerebrocardiac syndromes. It is difficult to treat cerebrocardiac syndromes. Based on conventional western drug therapy, our hospital applies Shenmai injection to treat patients with severe hypertensive ICH and cerebrocardiac syndromes, which has achieved outstanding therapeutic effect. Results are summarized in the following test.

\section{Methodology}

\subsection{Research Objects}

A total of 71 patients with severe hypertensive ICH and cerebrocardiac syndromes in the Neurosurgery Department of our hospital were selected and divided into the control group and the experimental group (Shenmai injection group) randomly. This study was approved by the Ethics Committee of the hospital and all patients have submitted a written informed consent.

\subsection{Inclusion and Exclusion Standards}

Inclusion standards: 1) history of hypertension, GCS score $<12$, aged between 50 - 85. 2) typical hypertensive ICH positions; 3 ) conforming to diagnosis standards of cerebrocardiac syndromes, including a series of symptoms of arrhythmia, myocardial ischemia, cardiac insufficiency and acute myocardial infarction, abnormal myocardial enzyme spectra and ECG changes.

Exclusion standards: 1) brain cavernous hemangioma, aneurysmal hemorrhage and tumor bleeding; 2) history of heart diseases like coronary heart disease and arrhythmia, extremely instable basic vital signs, severe heart, lung, liver and kidney diseases; 3) having allergies or serious adverse reactions to ingredients and accessories of Shenmai injection, having family allergy history to drugs and allergic physiques.

\subsection{Treatment Methods}

Both groups adopt conventional treatments, including dehydration-based reduc- 
tion of intracranial pressure, anti-infection and nutrition support. Patients with severe arrhythmia were administrated by propranolol and patients who have complications of heart failure were administrated by deslanoside and dobutamine. Surgical treatment was adopted to patients who had indications of operation. Based on the conventional treatment, the experimental group was provided with Shenmai injection (Zhengda Qingchunbao Group) additionally. Shenmai injection (50 ml) was added into $250 \mathrm{ml} 5 \%$ glucose injection, which was given through intravenous drip once every day, 2 weeks continuously.

\subsection{Index Observation Test}

Both groups attended in the conventional vital sign and electrocardiogram monitoring. At $6 \mathrm{~d}$ of hospitalization, myocardial enzyme spectra and troponin were tested. Moreover, myocardial enzyme spectra, including aspartic aminotransferase (AST), lactate dehydrogenase (LDH), creatine kinase (CK) and isozymes (CK-MB), were reexamined at $3 \mathrm{~d}, 7 \mathrm{~d}$ and $14 \mathrm{~d}$. All patients had $24 \mathrm{~h}$ electrocardiogram monitoring immediately after the hospitalization and ECG examination was performed upon the discovery of any anomalies. ECG examinations and GCS scores were performed at $7 \mathrm{~d}$ and $14 \mathrm{~d}$ after the hospitalization.

\subsection{Statistical Process}

The Statistical Program for Social Sciences software18.0 (SPSS 18.0) was applied. Measurement data was expressed by mean \pm standard deviation. The inter-group comparison used the two independent sample t-test and intragroup comparison before and after the treatment used the pairwise t-test. Inter-group comparison of enumeration data used the $\mathrm{x} 2$ test. $\mathrm{P}<0.05$ indicates that the difference has statistical significance.

\section{Results}

A total of 72 patients with severe hypertensive ICH who have been hospitalized in our hospital from January, 2020 to December, 2020 were chosen and divided into the control group and experimental group (Shenmai injection group) randomly, 36 cases in each group. After exclusion of patients, the control group had 31 cases and the experimental group had 30 cases. The control group was composed of 18 males and 14 females who were aged between $51-81$, averaging at $(63.81 \pm 9.32)$ years old. The experimental group had 17 males and 13 females who were aged between $50-79$, averaging at $(64.84 \pm 9.41)$ years old. Two groups have no significant differences in term of general conditions, CT scanning of bleeding position and volume of bleeding $(\mathrm{P}>0.05)$. This research scheme was approved by the Ethics Committee of our hospital and all patients signed the informed consent.

Myocardial enzyme indexes of two groups at $3 \mathrm{~d}, 7 \mathrm{~d}$ and $14 \mathrm{~d}$ were observed (Table 1). Myocardial enzyme index of the experimental group was improved quickly and significantly. Myocardial enzyme data of two groups at $3 \mathrm{~d}, 7 \mathrm{~d}$ and 
$14 \mathrm{~d}$ were $\mathrm{P}<0.05$. According to the electrocardiographic examinations results (Table 2), ECG anomalies of the experimental group at $7 \mathrm{~d}$ and $14 \mathrm{~d}$ were also improved quickly $(\mathrm{P}<0.05)$. It can be seen from Table 3 that there's no evident anomalies in GCS scores of two groups at 7d, but GCS score of the experimental group at $14 \mathrm{~d}$ of the medication was significantly higher than that of the control group $(\mathrm{P}<0.05)$.

\section{Discussions}

As a sever acute disease with high incidence rate in clinics, hypertensive ICH has clinical characteristics of sudden onset, quick progression, high fatality rate and poor prognosis. In China, the number of patients with $\mathrm{ICH}$ is increasing in the continuous aging process. Cardiovascular and cerebrovascular diseases have become the top 1 cause of death. Cerebrocardiac syndromes refer to a series of signs, symptoms, myocardial enzyme spectra anomalies and ECG changes similar with arrhythmia, myocardial ischemia, cardiac insufficiency and acute myocardial infarction caused by acute cerebrovascular accidents. Clinical symptoms of patients with cerebrocardiac syndromes include quick progress, sudden onset and high mortality. Cerebrocardiac syndromes will threaten life safety of patients significantly if they are not treated timely.

Patients with acute cerebrovascular diseases often are accompanied with cerebrocardiac syndromes. It is reported that the incidence rate of acute cerebrovascular

Table 1. Myocardial enzyme spectra test results.

\begin{tabular}{cccccc}
\hline & (cases) & CK (U/L) & CK-MB (U/L) & AST (U/L) & LDH (U/L) \\
\hline \multirow{2}{*}{ Experimental group } & $3 \mathrm{~d}$ & $546 \pm 87$ & $182 \pm 59$ & $73 \pm 23$ & $443 \pm 79$ \\
$\mathrm{~N}=30$ & $7 \mathrm{~d}$ & $234 \pm 63$ & $81 \pm 32$ & $43 \pm 12$ & $279 \pm 43$ \\
& $14 \mathrm{~d}$ & $82 \pm 23$ & $36 \pm 23$ & $28 \pm 6$ & $84 \pm 23$ \\
Control group & $3 \mathrm{~d}$ & $732 \pm 123$ & $232 \pm 89$ & $121 \pm 48$ & $632 \pm 112$ \\
$\mathrm{~N}=32$ & $7 \mathrm{~d}$ & $432 \pm 82$ & $134 \pm 79$ & $68 \pm 17$ & $354 \pm 67$ \\
& $14 \mathrm{~d}$ & $133 \pm 35$ & $56 \pm 28$ & $33 \pm 8$ & $112 \pm 42$ \\
\hline
\end{tabular}

Table 2. ECG examination results.

\begin{tabular}{cccc}
\hline & (cases) & $\begin{array}{c}\text { EKG recovers to } \\
\text { normal at 7d (cases) }\end{array}$ & $\begin{array}{c}\text { EKG recovers to } \\
\text { normal at 14d (cases) }\end{array}$ \\
\hline Experimental group & 30 & 18 & 28 \\
Control group & 32 & 11 & 21 \\
P-value & & $<0.05$ & $<0.05$ \\
\hline
\end{tabular}

Table 3. GCS scores.

\begin{tabular}{cccc}
\hline & (Cases) & GCS score at 7d & GCS score at 14d \\
\hline Experimental group & 30 & $10.56 \pm 1.43$ & $12.89 \pm 1.53$ \\
Control group & 32 & $10.86 \pm 1.53$ & $11.36 \pm 1.65$ \\
P value & & $>0.05$ & $<0.05$ \\
\hline
\end{tabular}


diseases is about $80 \%$. Clinically, the rate of missed diagnosis and misdiagnosis rate are relatively high. The superposition of acute cerebrovascular diseases and cerebrocardiac syndromes can aggravate the disease condition of patients, increase difficulties in treatment and affect prognosis significantly [1]-[6]. Treatment of severe hypertensive ICH must consider general circulation and brain circulation simultaneously. The clinical applications of Shenmai injection give us a good choice to realize brain-heart simultaneous treatment, address symptoms and root causes and improve prognosis.

Shenmai injection was derived from Shendong decoction in the Zhen Yin Mai Zhi. It is mainly prepared by Ginseng and Radix Ophiopogonis. It can nourish Yin and generate body fluid, tonify Qi and activate pulses. Shenmai injection is applicable to various shocks. It can excite interrenal system, increase elimination of various pathologic substances of the reticuloendothelial system (RES) during stroke, improve blood supply of important organs like heart, liver and brain, improve micro-circulation and anti-coagulation, strengthen heart and increase pressure, improve coronary flow, increase resistance to anoxia of body, decrease myocardial oxygen consumption, protect and repair myocardial cells and has certain anti-arrhythmia. Clinically, Shenmai injection is mainly applied to treat cardiovascular and cerebrovascular diseases [7].

According to modern pharmacological research, Shenmai injection can: 1) eliminate harmful substances like oxygen free radicals and proto-oncogene, inhibit calcium overload, improve blood circulation, promote synthesis of proteins and neurotransmitter in brains, and stop cells to initiate suicide program; 2) decrease apoptosis [8]; 3) inhibit endogenous nitric oxide synthase (NOS), relieve vasogenic edema, relieve inflammatory reactions, promote hematoma absorption, and strengthen immunity of the body [9] [10]. Shenmai injection can lower expression of tumor necrosis factor-a (TNF-A) in ischemic zone surrounding the hematoma, and inhibit expression of interleukin (IL-6) and proteolytic enzymes. Besides, it can increase expression of HSP70 in ischemic zone surrounding the hematoma and stop thrombosis and endothelia injury caused by inflammations, thus enabling to protect nerves [11].

Shenmai injection can protect myocardial damages of patients after carido-pulmonary resuscitation to some extent. The modern pharmacological studies have found that the relevant mechanism might be related with anti-lipid peroxidation, increased activity of superoxide dismutase of cardiac muscle tissues, decreased levels of $\mathrm{CK}-\mathrm{MB}$, cardiac troponin $\mathrm{T}$ (cTnT) and N-terminal B-type natriuretic peptide precursor (NT-proBNP), and improved left ventricular ejection fraction (LVEF), stroke volume (SV) and cardiac output (CO) [12] [13] [14] [15].

Myocardial enzyme indexes of two groups at $3 \mathrm{~d}, 7 \mathrm{~d}$ and $14 \mathrm{~d}$ were observed. It found that the myocardial enzyme index of the experimental group was improved quickly and significantly. Myocardial enzyme data of two groups at $3 \mathrm{~d}$, $7 \mathrm{~d}$ and $14 \mathrm{~d}$ were $\mathrm{P}<0.05$. According to the electrocardiographic examinations results, ECG anomalies of the experimental group at $7 \mathrm{~d}$ and $14 \mathrm{~d}$ were also im- 
proved quickly $(\mathrm{P}<0.05)$. There are no evident anomalies in GCS scores of two groups at $7 \mathrm{~d}$, but GCS score of the experimental group at $14 \mathrm{~d}$ of the medication was significantly higher than that of the control group $(\mathrm{P}<0.05)$.

According to pharmacological and experimental studies, Shenmai injection has certain therapeutic effects to cerebral injuries and myocardial damages. It can improve microcirculation disturbance surrounding the hematoma and accelerate absorption of hematoma, which are beneficial to recovery of neurological functions and improve clinical therapeutic effect of rehabilitation patients.

On one hand, Shenmai injection to patients with severe hypertensive ICH and cerebrocardiac syndromes in the early stage can make full use of essence of Traditional Chinese Medicine. On the other hand, it can treat brain and heart diseases simultaneously, and realize the goal of addressing both symptoms and root causes. This is expected to improve prognosis of patients with severe hypertensive ICH and it shall be further studied.

\section{Fund-Supported Projects}

Zhejiang Medical Association Clinical research fund projects 2019ZYC-A157.

Shaoxing Health Science and technology plan 2017CX025.

Zhejiang Health Development Foundation 2019ZD059.

Zhejiang Health Development Foundation 2019ZA127.

\section{Conflicts of Interest}

The authors declare no conflicts of interest regarding the publication of this paper.

\section{References}

[1] Hu, Y. and Zhang, X. (2019) Clinical Characteristics of Acute Stroke Accompanied with Cerebrocardiac Syndromes. China Modern Medicine, 26, 102-104.

[2] Liu, S.T. (2019) Clinical Observations of Acute Stroke Accompanied with Cerebrocardiac Syndromes. Chinese Remedies \& Clinics, 19, 610-611.

[3] Wei, P.F. (2015) Clinical Analysis 50 Cases of Intracerebral Hemorrhage Accompanied with Cerebrocardiac Syndromes. Chinese Journal of Practical Neurological Diseases, No. 16, 71-72.

[4] Wei, J., Wang, H.L., Zhao, H.M., Zhou, J.T., Zhang, W., Xu, Y.Q. and Yu, X. (2013) Clinical Analysis of Intracerebral Hemorrhage Accompanied with Cerebrocardiac Syndromes. Chinese Journal of Gerontology, 33, 444-445.

[5] Wang, H.Y. and Wang, X. (2011) Clinical Review of 46 Cases of Acute Cerebrovascular Accidents Accompanied with Cerebrocardiac Syndromes. Journal of Jilin Medicine, 32, 4419-4420.

[6] Li, X.X. (2009) Cerebrocardiac Syndromes in the Acute Attack Stage of Intracerebral Hemorrhage. Journal of Chongqing Medicine, 38, 2127-2128.

[7] Sun, J.M. (2014) Research Progresses on Immunization of Shenmai Injection. Medical Front, No. 12, 52-53.

[8] Lei, J.S., Luo, L., Chen, L. and Wang, C.Q. (2008) Effects of Shenmai Injection on 
Cell Apoptosis Surrounding Hematoma after Intracerebral Hemorrhage of Rats. Journal of Hunan University of Chinese Medicine, 28, 29-31.

[9] Wang, Q.R., Wang, L.F. and Shen, H.P. (2011) Effects of Panax Notoginsenosides and Shenmai Injection on Posterior Brain Edema and Behavioral Changes of Rats with Intracerebral Hemorrhage. Chinese Journal of Gerontology, 31, 288-290.

[10] Li, Y.J., Qian, W.J., Zhang, Y.K., et al. (2016) Effects of Shenmai Injection on Transforming Growth Factor $\beta 1$ and Neurological Function of Patients with Intracerebral Hemorrhage. Chinese Journal of Practical Neurological Diseases, 19, 120-121.

[11] Zhang, J.J. (2015) Clinical Research Progress of Shenmai Injection. Chinese Journal of Modern Drug Application, 9, 266-267.

[12] He, C., Long, L., Du, Q.S., Feng, J., Liu, C.X. and Wang, X.L. (2019) Effects of Shenmai Injection in Patients with Sepsis Myocardial Damages. Journal of Hebei Medical University, 40, 785-788.

[13] Chen, F., Jia, Y.H. and Liu, J. (2017) Clinical Observation of Shenmai Injection Treatment in Infectious Shock Combined with Myocardial Damages. Chinese Journal of Traditional Medical Science and Technology, 24, 624-625, 652.

[14] Wang, J.X., Zhang, K.T., Zhen, X.L., Jiang, T. and Han, P. (2017) Clinical Observations of Therapeutic Effects of Shenmai Injection on Myocardial Damages of Patients with Cardio-Pulmonary Resuscitation. Chinese Journal of Integrated Traditional and Western Medicine in Intensive and Critical Care, 24, 598-601, 621.

[15] Xu, T., Wang, W.T. and Lu, N.Q. (2009) Protective Effects of Shenmai Injection on Myocardial Damages of Rats. Aerospace Medicine, 20, 7-8. 